\title{
FORMULAÇÃO DE PÓ FLUXANTE A PARTIR DE RESÍDUOS DE MÁRMORE E GRANITO*
}

\author{
Letícia Miranda Alves ${ }^{1}$ \\ Heitor Cristo Clem de Oliveira ${ }^{1}$ \\ Hiury Voltz ${ }^{1}$ \\ Cássio Santos de Carvalho ${ }^{2}$ \\ José Roberto de Oliveira ${ }^{3}$ \\ Estéfano Aparecido Vieira ${ }^{3}$
}

\section{Resumo}

Os pós fluxantes têm como base o sistema $\mathrm{CaO}-\mathrm{SiO}_{2}-\mathrm{Al}_{2} \mathrm{O}_{3}-\mathrm{Na}_{2} \mathrm{O}-\mathrm{CaF} 2$ sendo que, em alguns casos, podem conter também $\mathrm{MgO}, \mathrm{Li}_{2} \mathrm{O}, \mathrm{K}_{2} \mathrm{O}$ e $\mathrm{B}_{2} \mathrm{O}_{3}$. Os resíduos de mármore e granito, oriundos dos processos de produção de rochas ornamentais, são passivos ambientais na região sudeste, sobretudo no estado do Espírito Santo. As composições destes resíduos são similares a dos pós fluxantes utilizados nos processos de lingotamento contínuo e portanto serão utilizados neste trabalho para formulação de novos fluxantes. Uma mistura de granito, pó de mármore e adições foi realizada com o objetivo de produzir um fluxante similar aos comerciais encontrados no mercado. O novo fluxante foi caracterizado por difração de Raios $\mathrm{X}$ antes e depois de passar pelo teste de susceptibilidade de formação de fases cristalinas ou "teste de Stollberg" modificado. Os resultados mostraram que existe a formação de fases similares às observadas em fluxantes comerciais. Avanços nesta área podem viabilizar economia de matéria-prima e a diminuição do impacto ambiental causado pelo mercado de rochas.

Palavras-chave: Lingotamento contínuo; Resíduo de mármore; Resíduo de granito; Pó fluxante.

\section{FORMULATION OF MOULD FLUX USING MARBLE AND GRANITE RESIDUE}

\section{Abstract}

The casting powders are based on $\mathrm{CaO}-\mathrm{SiO}_{2}-\mathrm{Al}_{2} \mathrm{O}_{3}-\mathrm{Na}_{2} \mathrm{O}-\mathrm{CaF} 2$ system and, in some cases, may also contain $\mathrm{MgO}, \mathrm{Li}_{2} \mathrm{O}, \mathrm{K}_{2} \mathrm{O}$ and $\mathrm{B}_{2} \mathrm{O}_{3}$. Marble and granite waste, originating from the ornamental production process, are environmental liabilities in the Southeast, especially in the state of Espírito Santo. The compositions of these wastes are similar to the mould flux used in continuous casting process and therefore will be used in this work for the formulation of new fluxes. A mixture of granite and marble dust plus additions was made to produce a similar commercial flux found in the market. The new flux was characterized by X-rays before and after of susceptibility test of crystalline phases formation. The results showed a formation of similar phases to that observed in commercial fluxes. Advances in this area can enable raw materials economy and the reduction of environmental impact caused by rocks market.

Keywords: Continuous casting; Marble residue; Granite residue; Mould flux.

1 Engenheiros Metalurgistas e Mestrandos em Engenharia Metalúrgica e de Materiais do Programa de Pós-Graduação em Engenharia Metalúrgica e de Materiais, PROPEMM, Vitória, ES, Brasil.

2 Tecnólogo em Rochas e Pós-Doutorando em Engenharia Metalúrgica e de Materiais do Programa de Pós-Graduação em Engenharia Metalúrgica e de Materiais, PROPEMM, Vitória, ES, Brasil

3 Engenheiro Metalurgista e Prof. Dr. do Instituto Federal do Espírito Santo, IFES, dos cursos de graduação e pós-graduação em Engenharia Metalúrgica e de Materiais, PROPEMM, Vitória, ES, Brasil. 


\section{INTRODUÇÃO}

O lingotamento contínuo é um processo altamente promissor e mais de 1 bilhão de toneladas de aço são lingotados por ano via este método [1]. Pode ser considerado, essencialmente, como um processo de transferência de calor no qual o metal líquido é transformado, via solidificação, em um produto sólido semi-acabado [2]. Neste processo está presente o molde, onde continuamente é adicionado o pó fluxante acima do aço líquido. Os pós fluxantes consistem de uma mistura de minerais que tem como base o sistema $\mathrm{CaO}-\mathrm{SiO}_{2}-\mathrm{Al}_{2} \mathrm{O}_{3}-\mathrm{Na}_{2} \mathrm{O}-\mathrm{CaF}_{2}$ que quando em contato com o aço líquido devem fundir e gerar uma escória líquida com características específicas para cada condição de lingotamento [3].

Os fluxantes influenciam diretamente nas condições de solidificação no lingotamento. Adicionado na superfície do aço no molde, este se funde e forma uma camada de escória líquida. Subsequentemente, essa escória se infiltra entre a "pele" do aço e o molde oscilante, criando um filme de escória que se solidifica em uma fase vítrea e outra cristalina. As propriedades desta escória estão associadas às principais funções do pó fluxante, ou seja, a lubrificação e a transferência de calor [4]. O fluxo de calor na horizontal é controlado pela espessura e natureza do filme de escória do fluxante formado entre a pele de aço solidificada e o molde de cobre. Além disso, no processo de lingotamento contínuo, o fluxo de calor é dependente do comportamento de cristalização do pó fluxante, ou seja, depende da temperatura de cristalização e dos tipos de fases cristalinas formadas. Portanto, é imprescindível que o fluxante possua propriedades de cristalização ideais para se obter o requerido fluxo de calor no lingotamento contínuo [5] combinado também com a viscosidade adequada.

De uma maneira geral, a formação da camada cristalina inibe a transferência de calor por radiação devido à opacidade desta fase. Portanto, um pó fluxante ao se cristalizar reduz sensivelmente o fluxo de calor da placa para o molde [6,7]. No caso do lingotamento de aços médio carbono $(0,06-0,18 \% \mathrm{C})$, estes são mais propensos a formarem trincas longitudinais devido à alta contração na passagem da fase " $\delta$ " para " $\gamma$ " e portanto escórias mais cristalinas são utilizadas neste caso, pois reduzem a transferência de calor placa/molde e amenizam a contração deste tipo de aço [3]. 0 aumento de cristalinidade causado pelo $\mathrm{CaO}$ não é novidade, pois é conhecido que um aumento de basicidade $\left(\% \mathrm{CaO} / \% \mathrm{SiO}_{2}\right)$ causa um aumento de cristalinidade [8]. Além disso, a porcentagem de cristalinidade também está relacionada com a quantidade de cuspidina formada e a ocorrência deste elemento ocorre em amostras que contém flúor [9].

Por outro lado, atualmente, grande parte dos processos industriais são fontes geradoras de resíduos na forma de gases, líquidos ou sólidos e causam grande degradação ambiental e, portanto, sem desenvolvimento sustentável. Os resíduos de mármore e granito são exemplos, e podem ser oriundos da extração do bloco, da serragem para enquadrá-los nas dimensões padronizadas, do processo de corte e de polimento, além dos finos da lavra e do beneficiamento. Na serragem, cerca de $20 \%$ a $30 \%$ do bloco é transformado em pó que não é aproveitado [10]. A produção brasileira de rochas ornamentais e de revestimento no ano de 2014 está apresentada na Tabela 1 e pode ser observado a grande quantidade de mármore e granito produzida, o que gera uma imensa quantidade de resíduos [11]. Estes resíduos têm grande similaridade de composição química se comparado a um pó fluxante comercial e, portanto, foram utilizados para a formulação de um novo pó fluxante. 
Tabela 1. Produção brasileira de rochas ornamentais e de revestimento obtidas no ano de 2014 [11].

\begin{tabular}{lcc}
\hline MATERIAL & PRODUÇÃO (t x 106) & PRODUÇÃO (\%) \\
\hline Granito e similares & 5,0 & $50 \%$ \\
Mármore e travertino & 2,1 & $21 \%$ \\
Ardósia & 0,5 & $5 \%$ \\
Quartzito foliado & 0,4 & $4 \%$ \\
Quartzito maciço & 0,9 & $9 \%$ \\
Pedra miracema & 0,2 & $2 \%$ \\
Outros & 1,0 & $10 \%$ \\
\hline
\end{tabular}

\section{MATERIAIS E MÉTODOS}

No presente estudo, foi proposto um novo pó fluxante com base em resíduos de mármore e granito e pequenas adições de fluorita e carbonato de sódio. Para fins comparativos, dois fluxantes comerciais foram também submetidos aos mesmos testes e análises, sendo um deles aplicável à aços peritéticos com forte tendência a formação de fases cristalinas e outro aplicável à aços baixo carbono cujo lingotamento possui baixa susceptibilidade de formação de trincas longitudinais.

\subsection{Formulação do Novo Pó Fluxante}

As Tabelas 2 e 3 apresentam a composição química dos fluxantes comerciais e dos materiais utilizados para formulação do novo fluxante, respectivamente. Análises químicas de orientação foram feitas usando-se microssonda por energia dispersiva (EDS) em um equipamento da Marca Oxford acoplado a um microscópio eletrônico de varredura (MEV), marca Zeiss Modelo Evo 10 pertencentes ao Laboratório de Microscopia Eletrônica de Varredura do IFES, campus Vitória. Devido às limitações deste tipo de equipamento, os resultados provavelmente não correspondem à real composição química das amostras e portanto há erros de até cerca de $5 \%$ para os elementos mais pesados podendo chegar a $20 \%$ para os elementos mais leves, dependendo também da quantidade presente de cada um.

Tabela 2. Composição química dos fluxantes comerciais peritético e baixo carbono obtida via balanço de massa a partir dos resultados do EDS.

\begin{tabular}{ccc}
\hline COMPOSTOS & $\begin{array}{c}\text { FLUXANTE PERITÉTICO } \\
\text { COMERCIAL (\%) }\end{array}$ & $\begin{array}{c}\text { FLUXANTE BAIXO CARBONO } \\
\text { COMERCIAL (\%) }\end{array}$ \\
\hline $\mathrm{CaO}$ & 30,5 & 25,1 \\
$\mathrm{SiO}_{2}$ & 22,9 & 30,4 \\
$\mathrm{Al}_{2} \mathrm{O}_{3}$ & 3,6 & 3,2 \\
$\mathrm{MgO}_{\mathrm{Na}}$ & 1,3 & 2,0 \\
$\mathrm{~K}_{2} \mathrm{O}$ & 4,8 & 8,9 \\
$\mathrm{Fe}_{2} \mathrm{O}_{3}$ & 0,2 & 0,2 \\
$\mathrm{~F}$ & 1,0 & 0,9 \\
$\mathrm{CaF}_{2}$ & 10,4 & 7,4 \\
$\mathrm{CO}_{2}$ & 0,0 & 0,0 \\
\hline TOTAL & 25,4 & 21,9 \\
\hline
\end{tabular}


Contudo, de forma orientativa, foi possível obter uma mistura para o fluxante proposto com composição química intermediária aos dois extremos conforme será mostrado adiante.

Tabela 3. Composição química da fluorita, do carbonato de sódio e dos resíduos de mármore e granito obtida via balanço de massa a partir dos resultados do EDS.

\begin{tabular}{ccccc} 
COMPOSTOS & $\begin{array}{c}\text { RESíDUO DE } \\
\text { MÁMORE (\%) }\end{array}$ & $\begin{array}{c}\text { RESÍDUO DE } \\
\text { GRANITO (\%) }\end{array}$ & FLUORITA (\%) & $\begin{array}{c}\text { CARBONATO } \\
\text { DE SÓDIO (\%) }\end{array}$ \\
\hline $\mathrm{CaO}$ & 39,1 & 0,9 & 0,0 & 0,0 \\
$\mathrm{SiO}_{2}$ & 2,0 & 66,0 & 2,1 & 0,0 \\
$\mathrm{Al}_{2} \mathrm{O}_{3}$ & 0,0 & 17,4 & 0,0 & 0,0 \\
$\mathrm{MgO}_{\mathrm{Na}} \mathrm{O}$ & 13,5 & 0,4 & 0,0 & 0,0 \\
$\mathrm{~K}_{2} \mathrm{O}$ & 0,0 & 2,9 & 0,0 & 58,2 \\
$\mathrm{Fe}_{2} \mathrm{O}_{3}$ & 0,0 & 6,4 & 0,0 & 0,0 \\
$\mathrm{~F}$ & 0,0 & 4,9 & 0,0 & 0,0 \\
$\mathrm{CaF}_{2}$ & 0,0 & 0,0 & 0,0 & 0,0 \\
$\mathrm{CO}_{2}$ & 0,0 & 0,0 & 95,1 & 0,0 \\
Impurezas & 45,4 & 1,2 & 2,8 & 41,3 \\
\hline TOTAL & 0,0 & 0,0 & 0,0 & 0,5 \\
\hline
\end{tabular}

A partir das composições químicas obtidas na Tabela 3, foram propostas faixas de composição química para a mistura estudada no presente trabalho. A Tabela 4 apresenta as devidas faixas do novo fluxante no qual buscou-se, por meio de balanço de massa, composições químicas próximas dos fluxantes comerciais.

Tabela 4. Faixas de composição química propostas para o novo fluxante obtidas via balanço de massa.

\begin{tabular}{ccccccccc}
$\mathrm{CaO}$ & $\mathrm{SiO}_{2}$ & $\mathrm{Al}_{2} \mathrm{O}_{3}$ & $\mathrm{MgO}$ & $\mathrm{Na}_{2} \mathrm{O}$ & $\mathrm{K}_{2} \mathrm{O}$ & $\mathrm{Fe}_{2} \mathrm{O}_{3}$ & $\mathrm{~F}$ & $\mathrm{CO}_{2}$ \\
$(\%)$ & $(\%)$ & $(\%)$ & $(\%)$ & $(\%)$ & $(\%)$ & $(\%)$ & $(\%)$ & $(\%)$ \\
\hline $18-23$ & $18-23$ & $3-6$ & $8-12$ & $3-6$ & $0-2$ & $0-2$ & $6-11$ & $23-28$ \\
\hline
\end{tabular}

Para se alcançar a composição química apresentada na tabela 4, foi utilizado cerca de $80 \%$ dos resíduos e cerca de $20 \%$ de carbonato de sódio e fluorita. Após a devida mistura dos materiais, o novo fluxante foi levado ao EDS onde foi obtida a composição química apresentada na tabela 5.

Tabela 5. Composição química do novo fluxante, obtida via balanço de massa a partir dos resultados do EDS.

\begin{tabular}{rcccccccc}
$\mathrm{CaO}$ & $\mathrm{SiO}_{2}$ & $\mathrm{Al}_{2} \mathrm{O}_{3}$ & $\mathrm{MgO}$ & $\mathrm{Na}_{2} \mathrm{O}$ & $\mathrm{K}_{2} \mathrm{O}$ & $\mathrm{Fe}_{2} \mathrm{O}_{3}$ & $\mathrm{~F}$ & $\mathrm{CO}_{2}$ \\
$(\%)$ & $(\%)$ & $(\%)$ & $(\%)$ & $(\%)$ & $(\%)$ & $(\%)$ & $(\%)$ & $(\%)$ \\
\hline 21,1 & 21,6 & 5,1 & 10,4 & 4,4 & 1,4 & 1,7 & 6,0 & 27,9 \\
\hline
\end{tabular}

Um importante parâmetro para se definir a qualidade de um fluxante é a sua basicidade. Este parâmetro repercute diretamente nas suas propriedades físicas e a mesma foi calculada por meio da equação 1 [12].

$$
B=\frac{\% \operatorname{CaO}+\% M g O}{\% \operatorname{SiO} 2}
$$

Onde \%i refere-se a porcentagem em peso de cada composto envolvido. 


\subsection{Teste de Cristalinidade}

Para verificar a qualidade do fluxante proposto, avaliou-se sua tendência à formação de fases cristalinas. Optou-se, então, por realizar o teste de Stollberg modificado que segundo Li et al [9] evita discrepâncias quando comparado com medidas feitas em campo. Neste caso, as amostras dos fluxantes comerciais e do novo fluxante proposto foram primeiramente descarbonetadas na temperatura de $650^{\circ} \mathrm{C}$ por 16 horas ao ar. Posteriormente, cerca de $30 \mathrm{~g}$ destas amostras foram colocadas em cadinhos de alumina para aquecimento a $1300^{\circ} \mathrm{C}$ por 20 minutos. E por fim, as amostras fundidas foram despejadas em um cadinho de aço inoxidável e aquecidas até $610^{\circ} \mathrm{C}$ por 20 minutos. Após esta última etapa, o cadinho foi inserido em água para resfriamento.

A descarbonetação é necessária para a eliminação dos gases voláteis e consequente diminuição da evaporação dos componentes voláteis na escória líquida de fluxante durante o aquecimento [6]. Já o aquecimento a $1300^{\circ} \mathrm{C}$ se deve ao fato de ser uma temperatura que garante a total fusão do fluxante [13] e o recozimento na temperatura de $610^{\circ} \mathrm{C}$ teve como objetivo a indução da cristalização do material [9]. A partir deste experimento foi possível analisar a cristalinidade das amostras por difração de Raios $X$. As fases presentes na nova mistura e nos pós fluxantes comerciais foram determinadas em um equipamento da marca Bruker Mod. D8 ADVANCED com goniômetro tipo Bragg-Brentano com ângulo $2 \theta$ variando de 10 até $100^{\circ}$, passo de $0,02^{\circ}$ com tempo de leitura de $1 \mathrm{~s}$ e rotação de $10 \mathrm{rpm}$. Este equipamento pertence ao Laboratório de Caracterização de Materiais do IFES, campus Vitória. As análises de difração de Raios X foram feitas nas amostras in natura, após descarbonetação na temperatura de $650^{\circ} \mathrm{C}$ e também após o teste de cristalinidade modificado.

\section{RESULTADOS E DISCUSSÃO}

O primeiro resultado relevante refere-se às basicidades dos fluxantes comercial baixo carbono, peritético e do novo formulado que após o cálculo apresentado na equação 1 resultaram em 0,89; 1,46 e 1,39 respectivamente. O uso de basicidade binária é comum para muitos autores, porém, devido à alta concentração de $\mathrm{MgO}$ no resíduo de mármore optou-se por considerar este composto. Em fluxantes comerciais, normalmente a concentração de $\mathrm{MgO}$ é baixa ou mesmo desprezível conforme pode ser constatado na Tabela 1. A utilização de óxido de magnésio ( $\mathrm{MgO}$ ) em detrimento do óxido de cálcio $(\mathrm{CaO})$ já foi prevista em uma patente [14] e parece trazer benefícios ao processo. Portanto, é viável o teor elevado de magnésio no novo fluxante o que auxilia indiretamente na basicidade.

O aumento de basicidade induz a formação de fases cristalinas nos fluxantes durante o processo de lingotamento [8]. Portanto, esta relação tende a ser maior para os fluxantes peritéticos, pois estes necessitam de um maior controle na transferência de calor no molde. E foi exatamente o encontrado, a basicidade para o fluxante comercial foi a maior dentre as amostras chegando a 1,46. Dependendo das condições do processo, alguns autores afirmam que a formação de uma escória com maior basicidade proporciona melhor absorção de inclusões [8]. O novo fluxante apresenta uma basicidade igual a 1,39 e portanto em um patamar bastante próximo ao do fluxante comercial peritético. 


\subsection{Fases Presentes}

A Figura 1 apresenta os resultados de difração de Raios $X$ dos fluxantes in natura. Pode-se perceber a presença dos compostos fluorita, quartzo e calcita em todos eles. Porém, a wollastonita não está presente no novo fluxante e provavelmente é devido a maior concentração de dolomita nesta amostra. Conforme é observado na Tabela 3, o resíduo de mármore é muito rico em $\mathrm{MgO}$. A presença de fluorita pode ser verificada em todas as amostras e apresentou-se coerente quanto a composição química apresentada nas Tabelas 2 e 5, pois o teor de flúor do fluxante comercial baixo carbono e do novo fluxante são próximos e já o teor de flúor do fluxante comercial peritético é mais alto, como pode ser visto nas intensidades dos picos de Raios $X$ apresentado na figura 1.

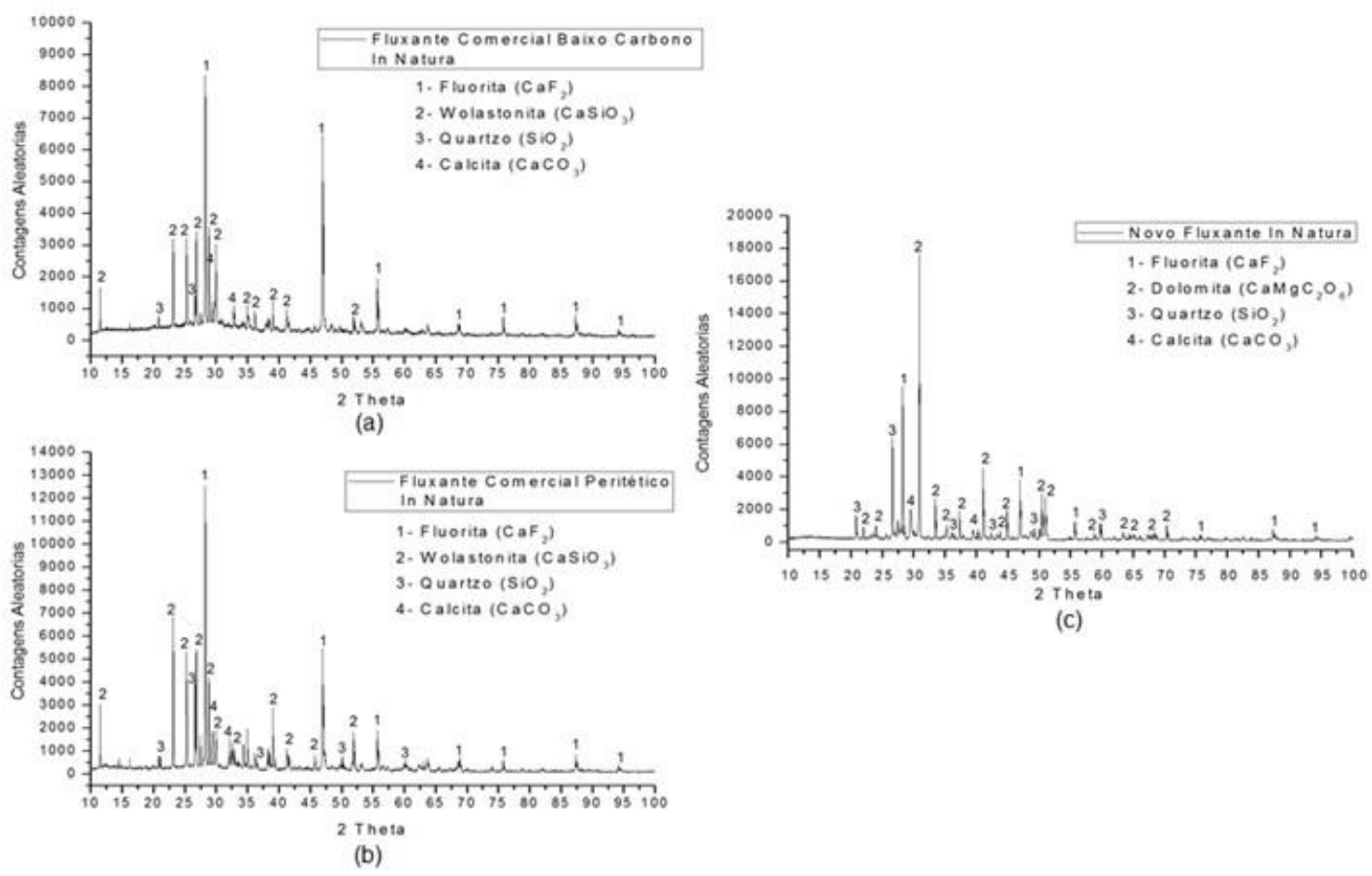

Figura 1. Análises de Raios $X$ das amostras in natura do (a) Fluxante baixo carbono comercial, (b) Fluxante peritético comercial e (c) Novo Fluxante.

Após a etapa de descarbonetação, procedeu-se novamente a análise por difração de Raios X e a Figura 2 mostra o resultado obtido. Foi identificado mais uma vez, no novo fluxante, a presença de $\mathrm{Mg}$ na forma de $\mathrm{MgO}$. Outro aspecto relevante é a formação de cuspidina $\left(\mathrm{Ca}_{4} \mathrm{~F}_{2} \mathrm{O}_{7} \mathrm{Si}_{2}\right)$ e nefelina $\left(\mathrm{NaAlSiO}_{4}\right)$ nos pós comerciais. O novo fluxante não apresentou a formação destas fases e acredita-se que este comportamento pode ser devido às diferentes proporções entre o $\mathrm{Na}_{2} \mathrm{O}, \mathrm{SiO}_{2}, \mathrm{CaO}, \mathrm{Al}_{2} \mathrm{O}_{3}$ e $\mathrm{CaF}_{2}$ presente em cada um deles. Neste trabalho, é difícil avaliar a influência de cada composto individualmente, já que temos diferentes concentrações nas misturas envolvidas. 


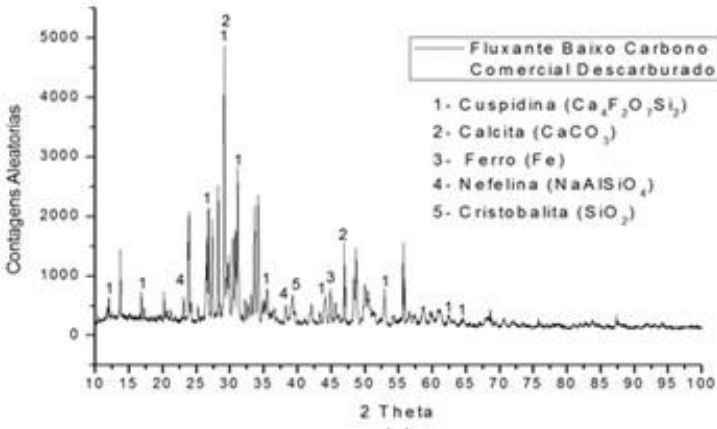

(a)

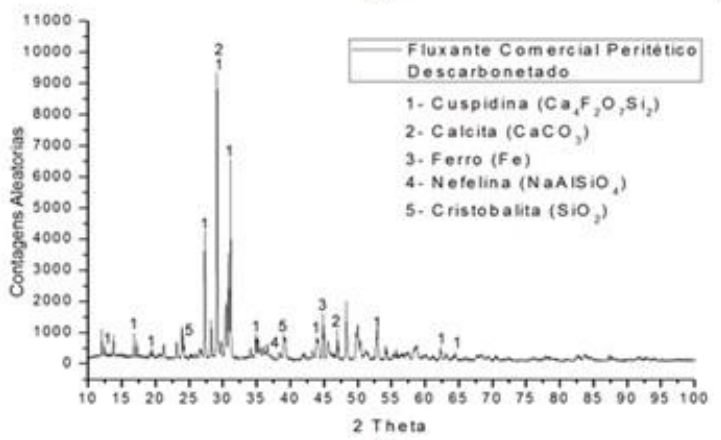

(b)

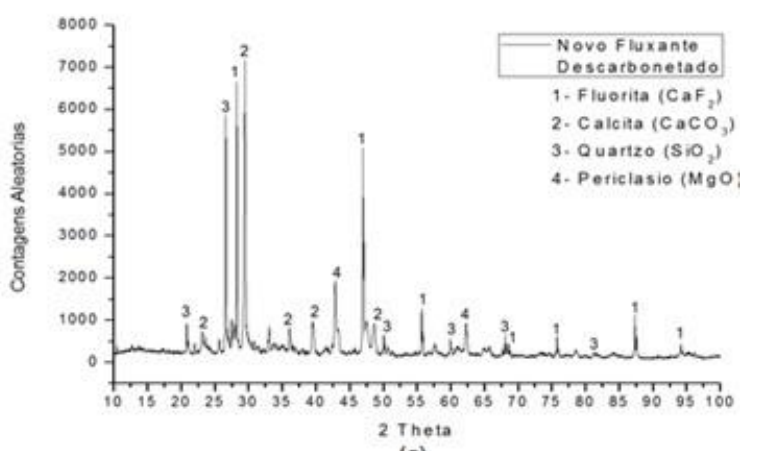

(c)

Figura 2. Análises de Raios $X$ após descarbonetação na temperatura de $650^{\circ} \mathrm{C}$ do (a) Fluxante baixo carbono comercial, (b) Fluxante peritético comercial e (c) Novo Fluxante.

Por fim, os resultados de difração de Raios $X$ das amostras submetidas ao teste de Stollberg modificado mostraram que o processo de aquecimento induziu a formação da cuspidina em todas elas, como pode ser visto na Figura 3. A cuspidina pode ser encontrada em todos fluxantes que contem flúor e a precipitação desta fase é a primeira a ser formada durante a têmpera dos fluxantes. Estas partículas contendo flúor podem agir como núcleos de cristalização. Portanto, a capacidade de formar fases cristalinas no processo de lingotamento pode ser relacionada com a quantidade de cuspidina na amostra obtida $[9,15]$.

Observa-se na figura 3 que a intensidade dos picos da cuspidina crescem em função da concentração de flúor $(F)$ mostrada nas Tabelas 2 e 5 . No fluxante baixo carbono comercial (Figura 3-a) a intensidade do pico está menor se comparado ao peritético (Figura 3-b), pois este último possui mais fluorita em sua composição. O novo fluxante apresentou uma intensidade de cuspidina próxima ao fluxante comercial baixo carbono devido ao fato de possuírem quantidades próximas de fluorita. Portanto, sugere-se um caminho para o desenvolvimento de um novo fluxante: para aplicações em liga peritéticas aumenta-se o flúor (F); e para ligas baixo carbono mantêm-se o teor de flúor no mesmo patamar (aproximadamente 6\%) e abaixa-se sua basicidade. Tendo em vista que fluxantes peritéticos devem possuir uma cristalinidade maior quando comparado com fluxantes baixo carbono [3,9], percebe-se que os difratogramas obtidos para os fluxantes comerciais estão de acordo com o esperado. Ao se fazer uma avaliação somente do ponto de vista da cristalinidade, o novo fluxante formulado parece estar mais semelhante ao fluxante comercial peritético, embora os difratogramas mostrem menor intensidade de cuspidina. No entanto, o teor deste elemento pode aumentar se houver um acréscimo na concentração de fluorita. O novo fluxante não mostra a formação de nefelina após o teste de Stollberg modificado e trata-se de uma fase secundária cristalina que geralmente aparece nos fluxantes para aços médio carbono [3,5]. Por fim, o novo fluxante apresentou um mineral chamado 
hibschite, proveniente do resíduo de granito, que possui teores elevado de $\mathrm{Al}_{2} \mathrm{O}_{3}$, favorecendo assim a formação de minerais a base de alumínio.

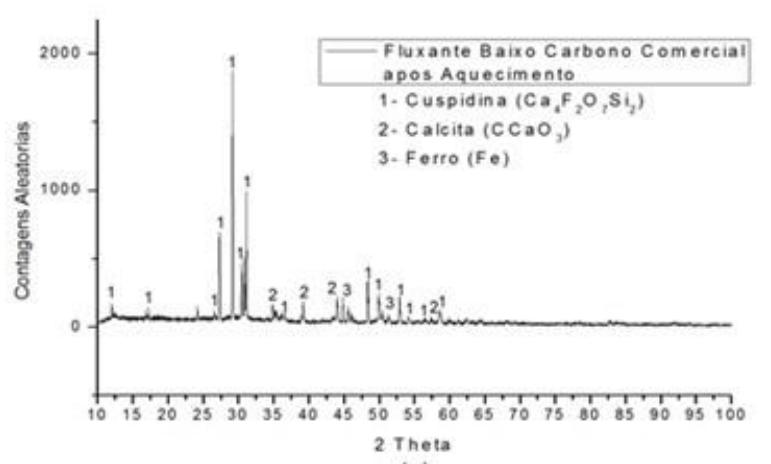

(a)
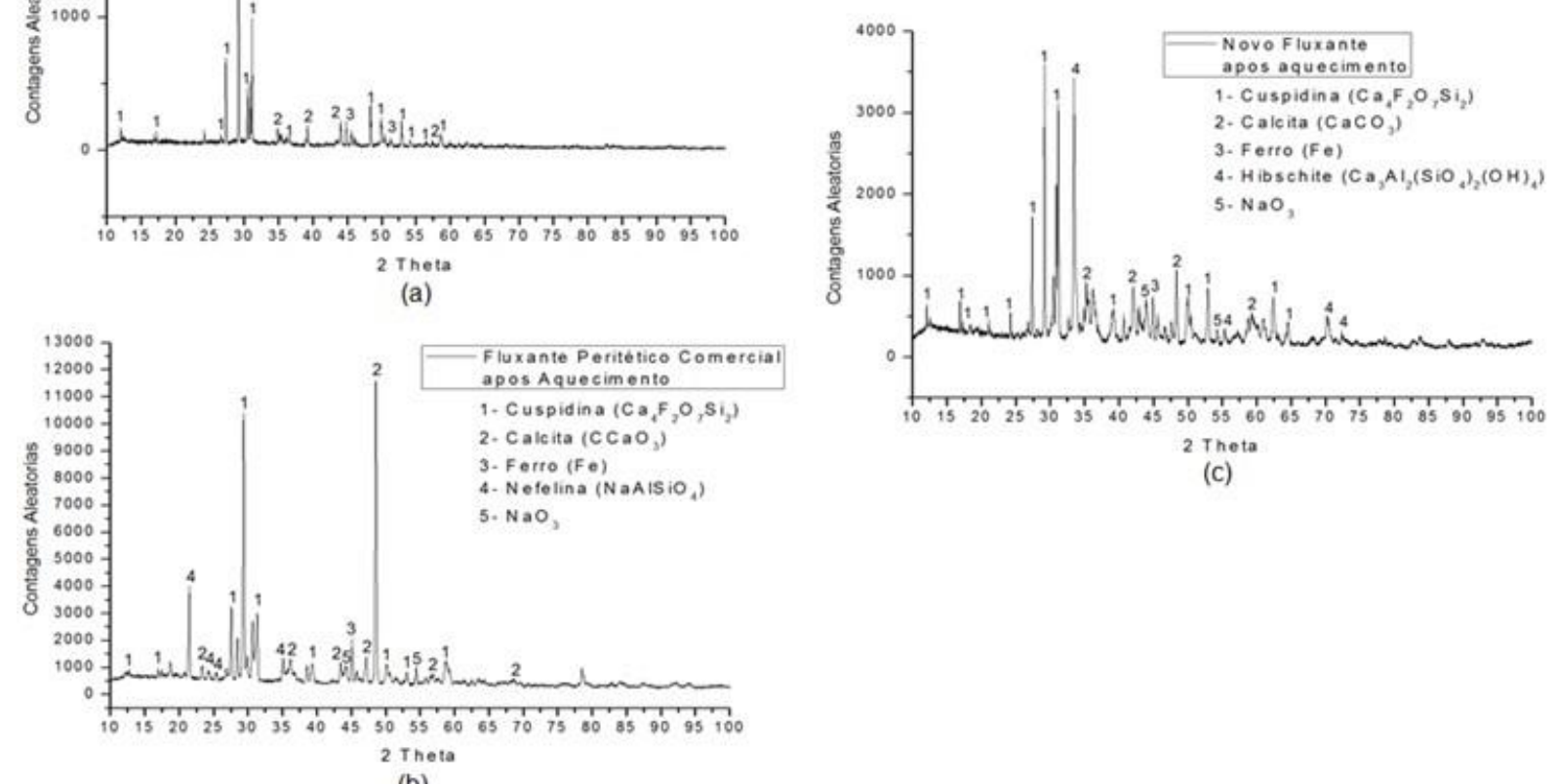

(b)

Figura 3. Análises de Raios $X$ após aquecimento do (a) Fluxante baixo carbono comercial, (b) Fluxante peritético comercial e (c) Novo Fluxante.

\subsection{Aspecto Visual das Amostras após Aquecimento}

Após o tratamento térmico apresentado no item 2.2, as amostras obtiveram a aparência da Figura 4. A Figura $4 \mathrm{a}, 4 \mathrm{~b}$ e $4 \mathrm{c}$ referem-se ao fluxante comercial baixo carbono, fluxante comercial peritérico e ao novo fluxante formulado, respectivamente.

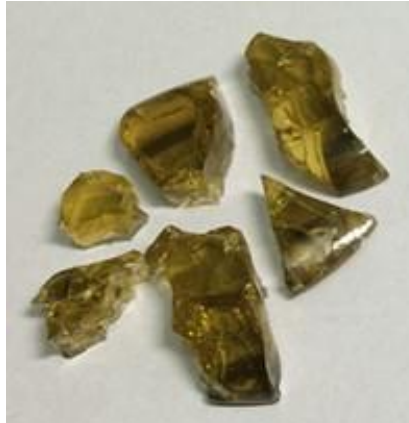

(a)

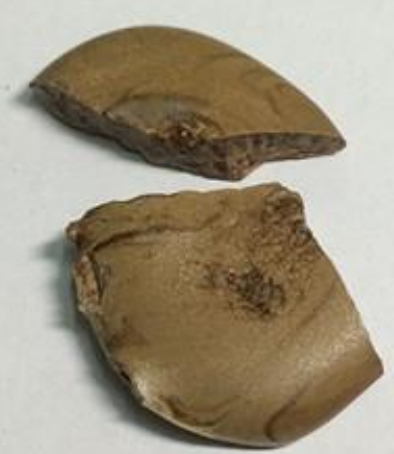

(b)

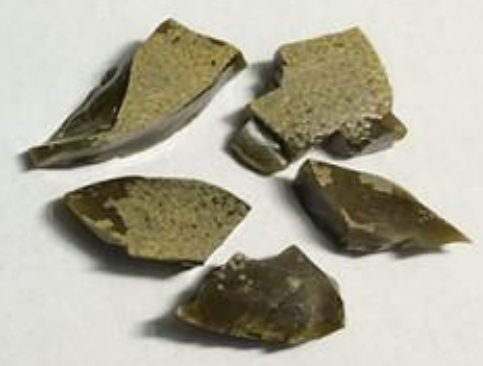

(c)

Figura 4. Amostras obtidas após o teste Stollberg modificado (a) Fluxante comercial baixo carbono, (b) Fluxante comercial peritético e (c) Novo fluxante.

O fluxante comercial peritético foi o que apresentou a aparência física de maior cristalinidade devido a sua inquestionável opacidade quando comparado com 0 
fluxante comercial baixo carbono. A cristalinidade maior induz uma menor taxa de transferência de calor através do filme. No caso do lingotamento de aços peritéticos a contração é muito elevada e provoca a formação de trincas e diante deste fato utilizase um fluxante mais cristalino (visualmente opaco) para redução da transferência de calor por radiação. O novo fluxante apresentou um aspecto próximo ao fluxante comercial peritético, mas para que sua cristalinidade possa alcançá-lo é viável um aumento do teor de flúor na sua composição.

Assim, conclui-se que a fabricação de fluxantes por meio da utilização de resíduos de rochas ornamentais mostra-se viável do ponto de vista de formação de fases. Contudo, outros estudos complementares relacionados a determinação da viscosidade, temperatura de cristalização, além de testes em campo precisam ser realizados a fim de consolidar esta proposta.

\section{CONCLUSÃo}

Diante da similaridade química e física entre os dois materiais, a utilização dos resíduos de mármore e granito para produção de fluxantes é um possível potencial de desenvolvimento. No entanto, deve-se ainda analisar outros parâmetros para que 0 desempenho no molde seja favorável, visto que é um material multifuncional e portanto outros aspectos devem ser investigados, como por exemplo viscosidade, lubrificação, temperatura de cristalização, capacidade de absorver inclusões, dentre outros.

\section{Agradecimentos}

Os autores agradecem ao Instituto Federal de Ciência e Tecnologia do Espírito Santo - IFES pelo apoio e agradecem também pelo apoio financeiro: À FAPES - Fundação de Amparo à Pesquisa do Espírito Santo; Ao CNPQ - Conselho Nacional de Desenvolvimento Científico e Tecnológico e a FINEP - Financiadora de Estudos e Projetos.

\section{REFERÊNCIAS}

1 Mills KC, Ramirez LP, Lee PD, Santillana B, Thomas BG, Morales R. Looking into continuous casting mould. Ironmaking and Steelmaking. 2014; 41(4): 242-249.

2 Garcia A, Spim JA, Santos CA, Cheung N. Lingotamento contínuo de aços. São Paulo: Associação Brasileira de Metalurgia e Materiais; 2006.

3 Li Z, Mills K, Bezerra MCC. Characteristics of mould flux films for casting MC and LC steels. In: XXXV Seminário de Fusão, Refino e Solidificação dos Metais. Salvador, Brasil; 2004.

4 Kromhout JA. Mould powder development for continuous casting of steel. Transactions of the Indian Institute of Metals. 2013; 66(5): 587-596.

$5 \mathrm{Seo} \mathrm{MD,} \mathrm{Shi} \mathrm{CB,} \mathrm{Cho} \mathrm{JW,} \mathrm{Kim} \mathrm{SH.} \mathrm{Crystallization} \mathrm{behaviors} \mathrm{of} \mathrm{CaO}-\mathrm{SiO}_{2}-\mathrm{Al}_{2} \mathrm{O}_{3}-\mathrm{Na}_{2} \mathrm{O}-$ $\mathrm{CaF}_{2}-\left(\mathrm{Li}_{2} \mathrm{O}-\mathrm{B}_{2} \mathrm{O}_{3}\right)$ mold fluxes. Metallurgical and Materials Transactions B. 2014; 45(5):1874-1886.

6 Görnerup M, Hayashi M, Däcker CA, Seetharaman S. Mould fluxes in continuous casting of steel - characterization and performance tuning. In: VII International Conference on Molten Slags Fluxes and Salts, The South African Institute of Mining and Metallurgy; 2004.

7 Cho JW, Emi T, Shibata H, Suzuki M. Heat transfer across mold flux film in mould during inicial solidification in continuous casting of steel. ISIJ International. 1998; 38(8): 834-842. 
$8 \quad$ Irving WR. Continuous casting of steel. The Institute of Materials, London, 1993.

9 Li Z, Thackray R, Mills KC. A test to determine crystallinity of mould fluxes. In: VII International Conference on Molten Slags, Fluxes and Salts, The South African Institute of Mining and Metallurgy; 2004.

10 Moura WA, Gonçalves J P, Leite RS. Utilização de resíduos de mármore e granito em argamassas de revestimento e confecção de lajotas para piso. Sitientibus. Feira de Santana. 2002; 26: 49-61.

11 ABIROCHAS, Balanço das exportações, importações, produção e consumo interno Brasileiro de rochas ornamentais. Associação Brasileira da Indústria de Rochas Ornamentais, 2014.

12 Sobrinho PJN, Tenório JAS. Recuperação de cromo presente em poeiras e lamas provenientes da fabricação de aço inoxidável - Influência da basicidade. Tecnologia em Metalurgia e Materiais. 2007; 4(2): 50-55.

13 Pereira MMSM, Nohara EL, Freitas SL, Ferreira GT, Moreira W C, Lima, MTD, Jung D. Pós fluxantes isentos de flúor para lingotamento contínuo de placas - Testes industriais. In: 45 Seminário de Aciaria - Internacional, Porto Alegre, Brazil, 2014.

14 Lechner HG, Pereira MMSM. Pó fluxante para lingotamento de aço. PI0704666-9 A2, INPI. 2007: 1-11.

15 Hanao M, Kawamoto M, Murakami T, Kikuchi H. Mold flux for high speed continuous casting of hypoperitectic steel slabs. Tetsu-to-hagane. 2002; 88(1): 23-28. 